\title{
Investigations of dust heating in M81, M83 and NGC 2403 with Herschel and Spitzer
}

\author{
George J. Bendo ${ }^{1}$ and the Herschel-SPIRE Local Galaxies \\ Guaranteed Time Programs
}

\author{
${ }^{1}$ UK ALMA Regional Centre Node, Jodrell Bank Centre for Astrophysics \\ School of Physics and Astronomy, University of Manchester \\ Oxford Road, Manchester M13 9PL, United Kingdom \\ email: george.bendo@manchester.ac.uk
}

\begin{abstract}
We use Herschel Space Observatory and Spitzer Space Telescope 70-500 $\mu \mathrm{m}$ data along with ground-based optical and near-infrared data to understand how dust heating in the nearby face-on spiral galaxies M81, M83, and NGC 2403 is affected by the starlight from all stars and by the radiation from star-forming regions. We find that $70 / 160 \mu \mathrm{m}$ flux density ratios tend to be more strongly influenced by star-forming regions. However, the 250/350 and $350 / 500 \mu \mathrm{m}$ micron flux density ratios are more strongly affected by the light from the total stellar populations, suggesting that the dust emission at $>250 \mu \mathrm{m}$ originates predominantly from a component that is colder than the dust seen at $<160 \mu \mathrm{m}$ and that is relatively unaffected by star formation activity. We conclude by discussing the implications of this for modelling the spectral energy distributions of both nearby and more distant galaxies and for using far-infrared dust emission to trace star formation.
\end{abstract}

Keywords. galaxies: ISM, infrared: general

\section{Introduction}

After the completion of the all-sky surveys by the Infrared Astronomical Satellite, astronomers have found conflicting evidence for the heating sources of the dust producing far-infrared emission in nearby galaxies. Some authors claimed that the dust was heated primarily by star formation (e.g. Devereux \& Young 1990; Buat \& Xu 1996) while others indicated that evolved stellar populations could heat the dust (e.g. Sauvage \& Thuan 1992; Walterbos \& Greenawalt 1996). This issue has become more important since the launch of the Herschel Space Observatory (Pilbratt et al. 2010). Herschel has been able to produce high signal-to-noise $>200 \mu \mathrm{m}$ images of both nearby and more distant galaxies, so it will be highly sensitive to colder dust that may have been missed by telescopes primarily observing at shorter wavelengths.

Several papers have been published on the sources of the heating for the dust emitting at Herschel wavelengths (e.g. Bendo et al. 2010, Rowan-Robinson et al. 2010, Boquien et al. 2011). We will focus on the results from Bendo et al. (2011) on the spiral galaxies M81, M83, and NGC 2403. Their analysis was based on comparing the infrared surface brightness ratios to $\mathrm{H} \alpha$ emission (used as a tracer of star formation) and $1.6 \mu \mathrm{m}$ emission (used as a tracer of the emission from the total stellar population). The infrared surface brightness ratios depend on dust heating, so they will appear correlated with the emission tracing the dust heating sources. 


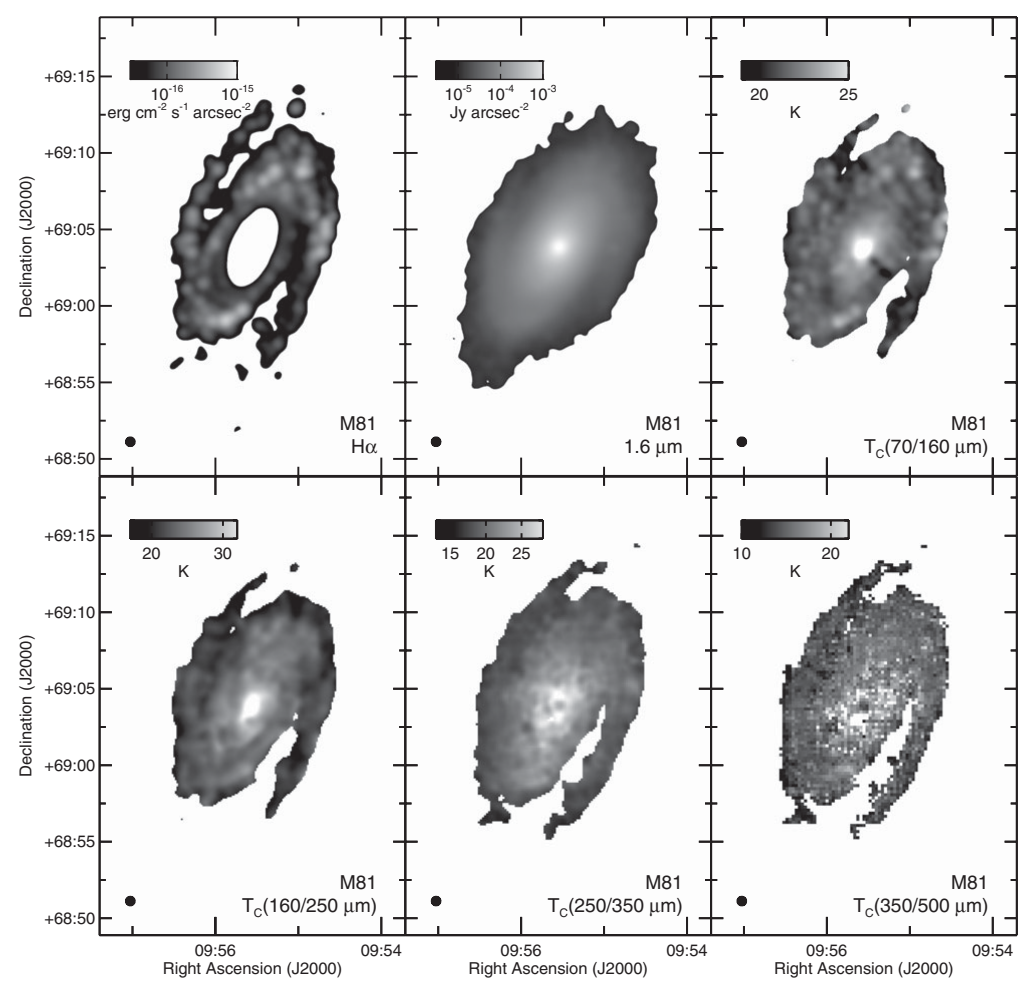

Figure 1. The colour temperature maps of $\mathrm{M} 81$ and $\mathrm{H} \alpha$ and $1.6 \mu \mathrm{m}$ images at the same resolution. The $70 \mu \mathrm{m}$ data are from Spitzer, the 160-500 $\mu \mathrm{m}$ data are from Herschel, the $\mathrm{H} \alpha$ data are from Boselli \& Gavazzi (2002), and the $1.6 \mu \mathrm{m}$ data are from Garrett et al. (2003). The colour temperatures are only shown for visualisation purposes; the quantitative analysis was performed on surface brightness ratios. All of these maps were created from data where the point spread function (PSF) was matched to the PSF of the $500 \mu \mathrm{m}$ data, which has a FWHM of $36^{\prime \prime}$ (shown by the circles in the lower left corner of each panel). Pixels where the signal was not detected at the $5 \sigma$ level in either the single band shown (in the case of the $\mathrm{H} \alpha$ and $1.6 \mu \mathrm{m}$ images) or in both bands (in the case of the colour temperature maps) were left blank (white). Each map is $30^{\prime} \times 20^{\prime}$, and north is up and east is left in each panel. See Bendo et al. (2011) for information on the creation of the colour temperature maps.

\section{Results}

Figures 1-3 show the data that were used in this comparison. Each galaxy yields similar but slightly different results. Details on the analysis, including quantitative analyses based on rebinned versions of these maps, are given by Bendo et al. (2011); we present a summarized version of the results here.

M81 has infrared surface brightness ratios that vary mostly as a function of the $1.6 \mu \mathrm{m}$ emission, which mainly depends on galactocentric radius. Except in the outer disc in the $70 / 160 \mu \mathrm{m}$ ratio map, very little local dust heating is seen. These results suggest that most of the dust seen at $160-500 \mu \mathrm{m}$ as well as some of the dust seen at $70 \mu \mathrm{m}$ is primarily heated by the total stellar population, including the evolved stellar population in the bulge.

In M83, both the star formation and the total stellar population trace the grand design spiral pattern of the galaxy, and so it is difficult to determine whether the infrared surface brightness ratios depend more on either the $\mathrm{H} \alpha$ or the $1.6 \mu \mathrm{m}$ emission. To disentangle the relative contributions of star-forming regions and the total stellar populations to dust 


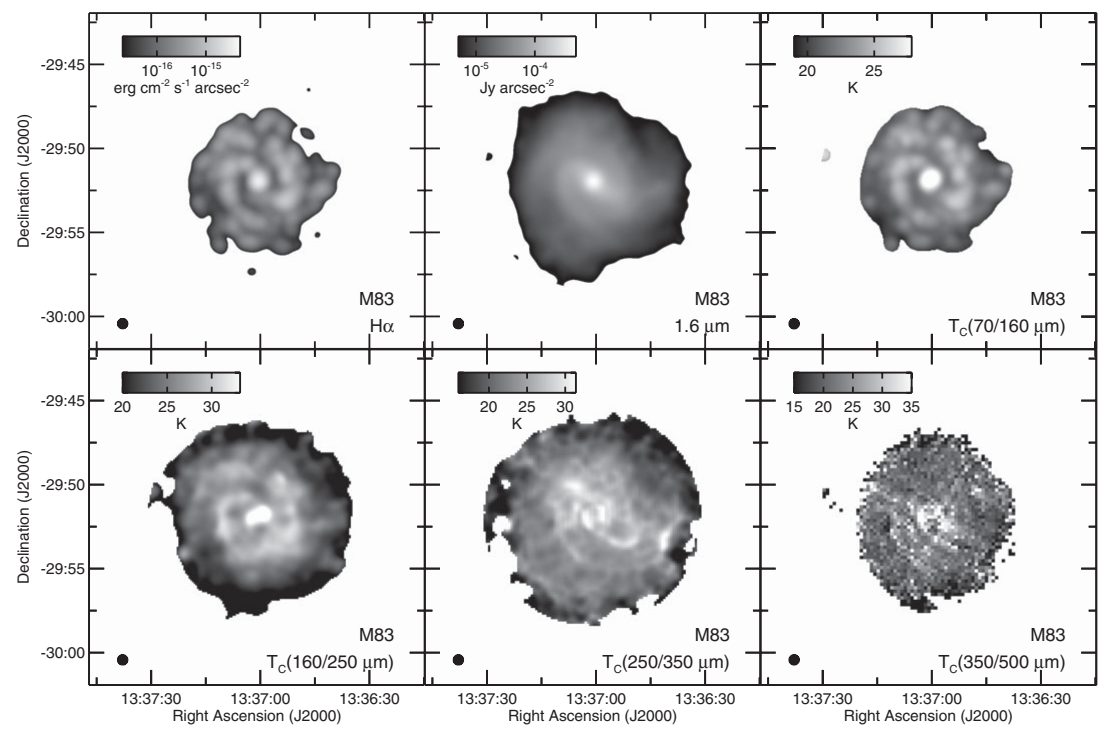

Figure 2. The colour temperature maps of $\mathrm{M} 83$ and $\mathrm{H} \alpha$ and $1.6 \mu \mathrm{m}$ images at the same resolution. The data sources are the same as for Figure 1 except that the $70 \mu \mathrm{m}$ data are from Herschel and the $\mathrm{H} \alpha$ data are from Meurer et al. (2006). Each map is $20^{\prime} \times 20^{\prime}$. See the caption of Figure 1 for additional information about the layout.

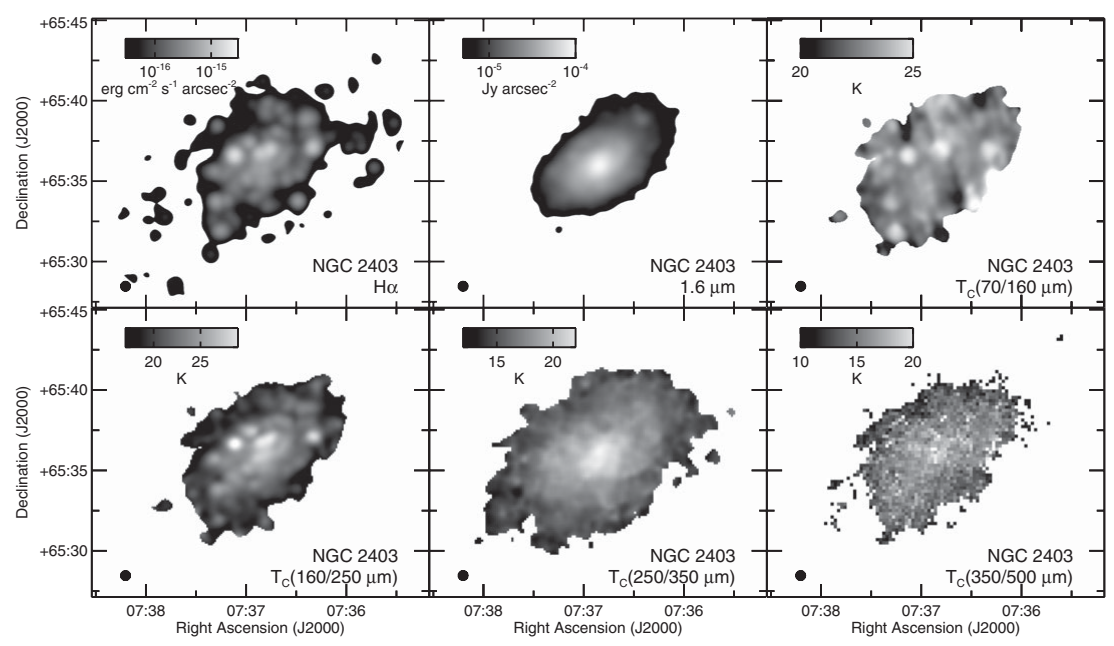

Figure 3. The colour temperature maps of NGC 2403 and $\mathrm{H} \alpha$ and $1.6 \mu \mathrm{m}$ images at the same resolution. The data sources are the same as for Figure 1. Each map is $21^{\prime} \times 18^{\prime}$. See the caption of Figure 1 for additional information about the layout.

heating as traced by any pair of wavelengths $\lambda_{1}$ and $\lambda_{2}$, we fit the data with

$$
\ln \left(\frac{I_{\nu}\left(\lambda_{1}\right)}{I_{\nu}\left(\lambda_{2}\right)}\right)=\alpha \ln \left(I(\mathrm{H} \alpha)+A_{1} I_{\nu}(1.6 \mu \mathrm{m})\right)+A_{2}
$$

and then examined the ratio of the $I(\mathrm{H} \alpha)$ and $A_{1} I_{\nu}(1.6 \mu \mathrm{m})$ terms. We found that the the $70 / 160$ and $160 / 250 \mu \mathrm{m}$ ratios primarily depend upon the $\mathrm{H} \alpha$ intensity while the $250 / 350$ and $350 / 500 \mu \mathrm{m}$ ratios primarily depend upon the $1.6 \mu \mathrm{m}$ surface brightness. This suggests that the emission from M83 at $>250 \mu \mathrm{m}$ originates from dust heated by the total stellar population. 
NGC 2403 shows notable differences in the dust heating as a function of wavelength. The $70 / 160$ and $160 / 250 \mu \mathrm{m}$ images show that the dust emission is clearly heated locally by the star-forming regions seen in the $\mathrm{H} \alpha$ image. However, the $250 / 350$ and $350 / 500 \mu \mathrm{m}$ ratios are more strongly correlated with the $1.6 \mu \mathrm{m}$ emission, again suggesting that the dust seen at $>250 \mu \mathrm{m}$ is heated by the total stellar population.

\section{Discussion}

Overall, these results show that dust emission in these three nearby spiral galaxies can be divided into a component that primarily emits at $<160 \mu \mathrm{m}$ that is heated by star-forming regions and a component that primarily emits at $>250 \mu \mathrm{m}$ that is primarily heated by the total stellar population, including evolved stars in the bulge and discs of these galaxies. This is generally consistent with the expectations from radiative transfer and dust emission models (e.g. Draine et al. 2007; Popescu et al. 2011). Despite this, the dust emission in individual wave bands between 70-500 $\mu \mathrm{m}$ observed from these galaxies is still correlated with $\mathrm{H} \alpha$ and other star formation tracers. This has also been found for $>100 \mu \mathrm{m}$ emission observed from other nearby galaxies as well (Boquien et al. 2010, 2011; Calzetti et al. 2010; Verley et al. 2010). These relations could arise even if the dust is not directly heated by the star-forming regions if the dust mass is correlated with star formation through the Schmidt law (e.g. Kennicutt 1998).

This has multiple implications. First of all, dust models and templates need to be adjusted to not only account for this very cold dust component but also to replicate the change in the colour variations as a function of wavlength. Dust extinction corrections that depend upon using infrared fluxes to estimate corrections need to account for dust heating by evolved stellar populations. Finally, far-infrared dust emission should be used cautiously as a star formation tracer, as dust emission may be related to star formation through the Schmidt law rather than through dust heating.

\section{References}

Bendo, G. J. et al. 2010, A\&A (Letters), 518, L65

Bendo, G. J. et al. 2011, in press (arXiv:1109.0237V1 [astro-ph])

Boquien, M. et al. 2010, A\&A (Letters), 518, L70

Boquien, M. et al. 2011, $A J, 142,111$

Boselli, A. \& Gavazzi, G. 2002, A\&A, 386, 124

Buat, V. \& Xu, C. 1996, A\&A, 306, 61

Calzetti, D. et al. 2010, ApJ, 714, 1256

Devereux, N. A. \& Young, J. S. 1990, ApJ (Letters), 350, L25

Draine, B. T. et al. 2007, ApJ, 663, 866

Kennicutt, R. C., Jr. 1998, ApJ, 498, 541

Jarrett, T. H., Chester, T., Cutri, R., Schneider, S. E., \& Huchra, J. P. 2003, AJ, 125, 525

Meurer, G. R. et al. 2006, ApJS, 165, 307

Pilbratt, G. et al. 2010, A\&A (Letters), 518, L1

Popescu, C. C., Tuffs, R. J., Dopita, M. A., Fischera, J., Kylafis, N. D., \& Madore, B. F. 2011, $A \& \mathcal{E} A, 527, \mathrm{~A} 109$

Rowan-Robinson, M. et al. 2010, MNRAS, 409, 2

Sauvage, M. \& Thuan, T. X. 1992, ApJ (Letters), 396, L69

Verley, S. et al. 2010, A\&A (Letters), 518, L68

Walterbos, R. A. M. \& Greenawalt B. 1996, ApJ, 460, 696 